\title{
A!
}

This is an electronic reprint of the original article.

This reprint may differ from the original in pagination and typographic detail.

Åström, Jan A.; Mäkinen, J. P.; Alava, M. J.; Timonen, J.

\section{Elasticity of Poissonian fiber networks}

Published in:

Physical Review E

DOI:

10.1103/PhysRevE.61.5550

Published: 01/05/2000

Document Version

Publisher's PDF, also known as Version of record

Please cite the original version:

Åström, J. A., Mäkinen, J. P., Alava, M. J., \& Timonen, J. (2000). Elasticity of Poissonian fiber networks.

Physical Review E, 61(5), 5550-5556. https://doi.org/10.1103/PhysRevE.61.5550

This material is protected by copyright and other intellectual property rights, and duplication or sale of all or part of any of the repository collections is not permitted, except that material may be duplicated by you for your research use or educational purposes in electronic or print form. You must obtain permission for any other use. Electronic or print copies may not be offered, whether for sale or otherwise to anyone who is not an authorised user. 


\title{
Elasticity of Poissonian fiber networks
}

\author{
J. A. Åström, ${ }^{1}$ J. P. Mäkinen, ${ }^{1}$ M. J. Alava, ${ }^{2}$ and J. Timonen ${ }^{1}$ \\ ${ }^{1}$ Department of Physics, University of Jyväskylä, P.O. Box 35, FIN-40351 Jyväskylä, Finland \\ ${ }^{2}$ Laboratory of Physics, Helsinki University of Technology, P.O. Box 1100, FIN-02015 TKK, Finland
}

(Received 22 November 1999)

\begin{abstract}
An effective-medium model is introduced for the elasticity of two-dimensional random fiber networks. These networks are commonly used as basic models of heterogeneous fibrous structures such as paper. Using the exact Poissonian statistics to describe the microscopic geometry of the network, the tensile modulus can be expressed by a single-parameter function. This parameter depends on the network density and fiber dimensions, which relate the macroscopic modulus to the relative importance of axial and bending deformations of the fibers. The model agrees well with simulation results and experimental findings. We also discuss the possible generalizations of the model.
\end{abstract}

PACS number(s): 62.20.Dc, 81.40.Jj

\section{INTRODUCTION}

The effective-medium model is a standard approach for estimating the elastic properties of inhomogeneous materials. One simply considers in the mean-field sense a representative volume element, and its response to mechanical perturbations. Such models are often successful if the structure of the material is simple enough. A more complicated approach is to consider variational bounds. They are dependent on the statistical properties of the medium through $N$-point correlation functions. There have been some efforts to describe various media via their three-point correlations since the latter relate directly to variational estimates of the elastic moduli [1]. Practical examples abound of materials that are structurally inherently heterogeneous (e.g., granular materials and fiber composites). For such materials the standard effectivemedium theory is doubtful, and there is no easy way to express the structural correlations in a useful manner.

In this paper we introduce an effective-medium model for the tensile stiffness of materials that are composed of randomly connected building blocks. For clarity we will not concentrate on the generic model, but on two-dimensional (2D) random networks of fibers. Such fiber networks are commonly used as a basic model of random fibrous materials such as paper or glass-fiber mats [2-8]. This application of the generic model gives us the possibility to test the approximative solution against numerical solutions. Extensions to other systems would depend on the geometry of these systems and on the elastic behavior of their building blocks.

The tensile modulus, or stiffness, of 2D random fiber networks obviously increases with increasing the areal mass density $\rho$ (coverage), which measures the number of fibers per unit area. Computer simulations (see e.g., $[4,5]$ ) reveal that the stiffness $\left(E_{e}\right)$ is asymptotically a linear function of $\rho$ such that

$$
E_{e}(\rho)=A\left(\rho-\rho_{0}\right),
$$

where $\rho_{0}$ and $A$ are constants to be determined. The former is related naturally to the geometrical percolation threshold $\rho_{c}$ since the network has to be geometrically connected in order to be able to bear load. Close to $\rho_{c}$ one finds a scaling regime similarly to other transport properties than elasticity [9] and only eventually, for high enough coverage $\rho \gg \rho_{c}$, the linear regime sets in. Such phenomena can also be reproduced experimentally in laboratory-made paper, showing that indeed $\rho_{0}>\rho_{c}$. The old effective-medium theory of Cox [11], the "Cox model," and the "shear-lag model" [12] that is based on it, have been used to explain Eq. (1). The Cox model assumes a shear-lag mechanism in which the stress of a matrix is gradually transferred to the fiber so that the stress is largest in the middle and reduced at the ends of the fibers. For low coverages the stress is not able to build up, and thus a lower stiffness arises [5,4]. The Cox-type models suffer, however, from several discrepancies. The value obtained for $\rho_{0}$ is much smaller than in reality, and $\rho_{0}$ is also predicted to depend on the average fiber length $L_{f}$. Also, the value of the modulus $A$ is reproduced, at most, qualitatively [10,5]. The failures of the shear-lag model result from the assumed stress-transfer mechanism that seems to be wrong [5-7], although the variation in the average stress with respect to fiber orientation is most likely correct $[4,5]$.

In this paper we demonstrate that the problems related to the Cox and shear-lag models can be eliminated with a reformulated effective-medium model. We use the same coarse-grained deformation field as in the Cox model. The mean-field approximation is, however, done at the discrete fiber-segment level since, in a fiber network, the stresses are transferred among segments at the fiber-to-fiber crossings. Thus we consider the segments as the basic building blocks of the system, and take into account their length distribution and assume that each segment is deformed in the energetically most favorable mode depending on its orientation and length. It is exactly this coupling between the geometry (segment length) and the deformation mode that makes the model nontrivial and improves on, e.g., the Cox model. The arguments used are not specific for random fiber networks and are, in fact, far more general. All random materials that can be viewed as sets of random points connected by elastic vector potentials at a rather low coordination number (as will be explained in detail below) are possible to model in a similar fashion. 
In Sec. II we define a random fiber network and describe the numerical techniques used. In Sec. III we outline the new effective-medium model. In the last two sections we compare the results of the modified effective-medium model with those of the numerical model, and finish with a summary.

\section{RANDOM FIBER NETWORKS}

A random fiber network is defined as a set of independently deposited short line segments on a two-dimensional plane. In the simplest case both the locations of the centers of mass and the orientation angles of the fibers are uniformly distributed throughout the plane, and the fiber length is constant. At a very low average number of fibers per unit area (i.e., at a low coverage) the fibers do not form a connected network. With increasing coverage the system reaches its geometrical percolation threshold at approximately $q_{c} \approx 5.7$ fibers per unit area (fiber length is unity) [13]. Another important threshold is the so called rigidity-percolation threshold $\left(q_{r c}\right)$. If the connections between the fiber-to-fiber bonds were central-force springs, a geometrically connected network would not necessarily have a nonzero stiffness. A central-force random fiber network consists of both rigid (triangles of segments) and nonrigid (more than three-sided polygons) substructures. The rigid substructures are, however, always elastically isolated as the coordination number of the bonds never exceeds 4 . Random spring networks have thus zero stiffness for any finite coverage [8,14], i.e., $q_{r c}$ $\rightarrow \infty$. This means that the stiffness of random fiber networks relies completely on the nonzero stiffness of all deformation modes of the individual fiber segments and not only of the axial mode.

In our numerical work the fibers are assumed to be rigidly bonded at each fiber-fiber crossing. This means that when the network is deformed, the angles between crossing fibers will remain constant and elastic strain will all be in the fiber segments between the bonds. There are three degrees of freedom for each fiber-fiber bond: horizontal displacement, vertical displacement, and rotation. The elastic interaction between two connected bonds is defined by a stiffness matrix. If the Young's modulus of a fiber segment of length $l$ is $E$, the fibers have a cross-sectional area $A$, and the moment of inertia of the cross section is $I$, then the stiffness matrix is given by

$\left|\begin{array}{cccccc}\frac{E A}{l} & 0 & 0 & -\frac{E A}{l} & 0 & 0 \\ 0 & \frac{12 E I}{l^{3}} & \frac{6 E I}{l^{2}} & 0 & -\frac{12 E I}{l^{3}} & \frac{6 E I}{l^{2}} \\ 0 & \frac{6 E I}{l^{2}} & \frac{4 E I}{l} & 0 & -\frac{6 E I}{l^{2}} & \frac{2 E I}{l} \\ -\frac{E A}{l} & 0 & 0 & \frac{E A}{l} & 0 & 0 \\ 0 & -\frac{12 E I}{l^{3}} & -\frac{6 E I}{l^{2}} & 0 & \frac{12 E I}{l^{3}} & -\frac{6 E I}{l^{2}} \\ 0 & \frac{6 E I}{l^{2}} & \frac{2 E I}{l} & 0 & -\frac{6 E I}{l^{2}} & \frac{4 E I}{l}\end{array}\right|$

which should be multiplied with the displacement vector $(X 1, Y 1, \Omega 1, X 2, Y 2, \Omega 2)$ to obtain the forces acting on the bonds at the segment ends. $X, Y$, and $\Omega$ correspond to horizontal, vertical, and rotation displacements, respectively, and the numbers 1 and 2 refer to the two bonds. In the following we assume for simplicity that the fibers have a square cross section of area $A=w^{2}$, which means that $I=w^{4} / 12$. The stiffness matrix is only valid when $w \ll l$. For short $l$ the bending stiffness $E w^{4} / l^{3}$ should, as a first approximation, be replaced by the shear modulus $E w^{2} /[2(1+\nu) l]$, where $\nu$ is the Poisson ratio.

In the numerical model we construct samples of random fiber networks according to the rules described above. We limit the size of the network to a rectangular surface of size $L_{x} L_{y}$, and the $x$ and $y$ coordinates of the centers of mass of the fibers are chosen from uniform distributions in the intervals $\left[-L_{f}, L_{x}+L_{f}\right]$ and $\left[0, L_{y}\right]$. The orientation of the fibers are chosen from a uniform distribution in the interval $[-\pi / 2, \pi / 2]$. We allow fibers to fall outside the box in order to avoid a lower coverage at the boundaries. The intersection points of the fibers are identified, and the stiffness matrix of the entire network is then constructed. This is done by rotations of the segment stiffness matrices by the in-plane angle of each segment and by adding the matrix elements corresponding to the same degree of freedom. Periodic boundary conditions are used in the vertical $y$ direction, and all fibers crossing the lines $x=0$ and $x=L_{f}$ are clamped at the crossing points. The clamped right boundary of the network is then forced to move a unit distance in the positive horizontal direction, while the left boundary is forced to remain at its original location. The equilibrium displacement of all bonds is calculated by the conjugate gradient method. The elastic stiffness of the network can thereafter be extracted. The equilibrium displacement of a small network is shown in Fig. 1.

\section{EFFECTIVE-MEDIUM MODEL}

In the effective-medium model we do not describe the fibers as distinct units in the network, nor the stress along fibers by, e.g., formulating a differential equation description of the stress-transfer such as in the Cox model. Such attempts would make the segment stresses correlated along the fibers with the reduced stress close to the fiber ends. Instead, we only consider the stress on the individual segments. We then use as input the fiber-segment-length probability distribution. This distribution is known for random (Poissonian) line networks, and a similar approach can obviously be applied in any disordered system in which the structural distribution of the constituents of the system is available. We combine this distribution with the argument that the fiber segments deform only in the energetically most favorable mode (the modes are bending, stretching, and shearing). In this way we can derive a "universal" stiffness curve of the random fiber network as a function of a single dimensionless parameter. This parameter is proportional to the dimensionless coverage multiplied by the ratio of the fiber width to fiber length (all fibers are assumed identical). When the width-to-length ratio vanishes the fiber segments behave as central-force springs which only "bend.' In the opposite limit only uniform-strain deformations are possible (i.e., shearing and stretching as opposed to bending). 


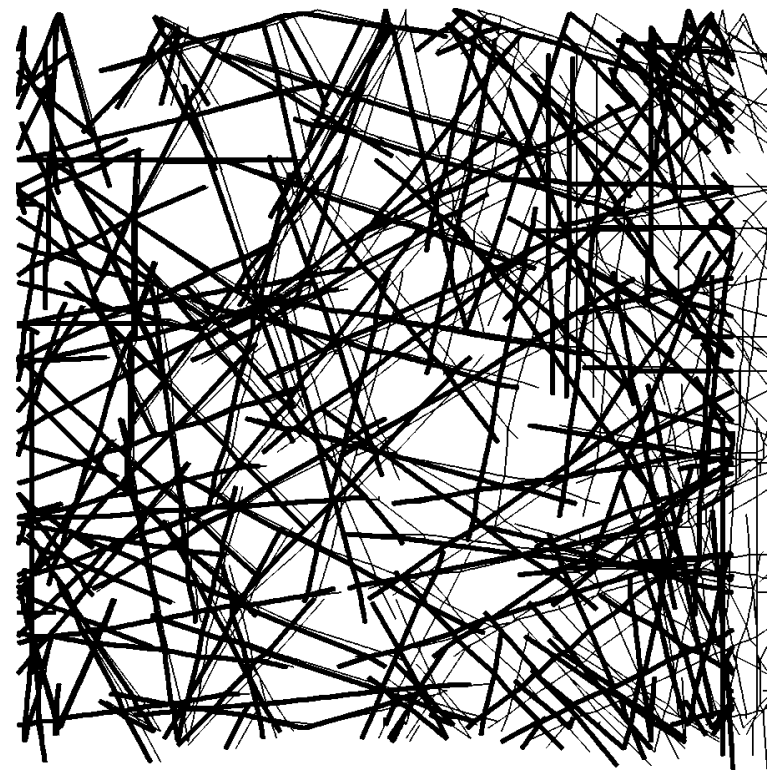

FIG. 1. A random fiber network with $q / q_{c}=4.0$. The thick lines show the unstrained network, and the thin lines the deformed network.

In quasistatic deformation the fiber segments are deformed such that there is force equilibrium at all fiber-fiber bonds. These points in the displacement space (i.e., displacements of bonds) also define the global minimum of the total elastic energy. This means that the fiber segments will, in general, be deformed in a way that offers the least elastic resistance. The segments can be deformed either by bending/ shearing or by stretching. As defined by the stiffness matrix above, the bending stiffness modulus is $E w^{4} / l^{3}$, the shear stiffness modulus is $E w^{2} /[2(1+\nu) l]$, and the elongation stiffness modulus is $E w^{2} / l$. Most important here is that the bending modulus depends on the ratio $w / l$ in a different way than the other two moduli. We now assume that a segment deforms only by bending if the bending modulus is smaller than both the shear and the elongation modulus, i.e., the segment has $l>l_{c} \equiv w \sqrt{2(1+\nu)}$. If $l<l_{c}$ the segments are assumed to deform by shearing and stretching. Notice that the goodness of this approximation is related to the lack of rigidity in the central-force fiber networks. Close to or above a rigidity threshold the segments would not be able to "choose" so freely their deformation modes based only on their own orientation and length. In random fiber networks this is a minor problem for the reasons outlined in Sec. II (triangles are not rigidly connected).

The above assumption is not enough to determine the stiffness of the network. We still need a way to quantify the magnitude of the displacements that take place. For this we choose the displacement field of the Cox model as it seems to well describe the displacements of the segments as a function of their orientation. That is, elongation of a segment is proportional to $\cos ^{2}(\theta)$, while bending and shear are proportional to $\cos (\theta) \sin (\theta)$, where $\theta$ is the angle between the direction of the external strain and the considered fiber segment. The effective-medium strain field does not include any rotations.

The final ingredient in our model is the segment-length distribution, which we need when using the segments as the basic building blocks of the systems. If all fibers in the network are deposited without correlations, the segment-length distribution is a one-dimensional Poisson process for which the length distribution is given by

$$
\sigma(l)=\frac{2 q}{\pi L_{f}} \exp \left(-\frac{2 q}{\pi L_{f}} l\right),
$$

where $L_{f}$ is the fiber length and $q$ is the dimensionless coverage. The average segment is $\hat{l}=\left(\pi L_{f} / 2 q\right)$. There is, of course, a cutoff in the distribution at $L_{f}$, but for large $q$ (i.e., for $q \gg q_{c}$, where $q_{c}$ is the geometrical percolation critical point) this can be neglected for simplicity.

The elastic energy of a fiber network is the sum of the elastic energy of the fiber segments. The elastic energy of a segment is its stiffness multiplied by the square of its displacement magnitude. We can thus calculate the elastic energy of a network by multiplying the stiffness of a particular deformation mode and the square of the deformation in that mode. We then multiply this with the dimension of the network and with the segment-length probability distribution, and then integrate everything over the range for which the particular mode is energetically the most favorable. The result including all three deformation modes is

$$
\begin{aligned}
W= & \frac{E w^{2}}{2} \epsilon_{x}^{2} q \frac{L_{x} L_{y}}{L_{f}} \int_{-\pi / 2}^{\pi / 2} \frac{\cos ^{4}(\theta)}{\pi} d \theta \int_{0}^{l_{c}} \frac{2 q}{\pi L_{f}} e^{\left[-2 q l /\left(\pi L_{f}\right)\right]} d l \\
& +\frac{G w^{2}}{2} \epsilon_{x}^{2} q \frac{L_{x} L_{y}}{L_{f}} \int_{-\pi / 2}^{\pi / 2} \frac{\cos ^{2}(\theta) \sin ^{2}(\theta)}{\pi} d \theta \\
& \times \int_{0}^{l_{c}} \frac{2 q}{\pi L_{f}} e^{\left[-2 q l /\left(\pi L_{f}\right)\right]} d l+\frac{E w^{4}}{2} \epsilon_{x}^{2} q \frac{L_{x} L_{y}}{L_{f}} \\
& \times \int_{-\pi / 2}^{\pi / 2} \frac{\cos ^{2}(\theta) \sin ^{2}(\theta)}{\pi} d \theta \int_{l_{c}}^{\infty} \frac{2 q}{\pi L_{f} l^{2}} e^{\left[-2 q l /\left(\pi L_{f}\right)\right]} d l
\end{aligned}
$$

where $\epsilon_{x}$ is the external strain, $L_{x} L_{y}$ is the dimension of the network, and $G=E /[2(1+\nu)]$. The essential parameter in Eq. (3) is $z \equiv 2 q l_{c} /\left(\pi L_{f}\right)$. The last integral is an exponential-integral function and cannot thus be expressed in terms of elementary functions $\left[E_{n}(z) \equiv \int_{1}^{\infty} e^{-z x} / x^{n} d x\right]$. By solving all the other integrals we obtain an expression for the elastic energy $(W)$ of the network as a function of $w$ and $q$. On the other hand, $W=(1 / 2) E_{e} \epsilon_{x}^{2} L_{x} L_{y}$, which means that we can get an expression for the stiffness of the network $\left(E_{e}\right)$ as a function of $w$ and $q$. The result for $E_{e}$ can be expressed in the form

$$
\begin{aligned}
E_{e}= & \frac{E w^{2} q}{8 L_{f}}\left[\left(\frac{2 q w}{\pi L_{f}}\right)^{2}\left(\frac{e^{-z}}{z}-E_{1}(z)\right)\right. \\
& \left.+\left(3+\frac{1}{2(1+\nu)}\right)\left(1-e^{-z}\right)\right]
\end{aligned}
$$

This equation is our first main result. We can test it by first considering the limit $w \rightarrow 0$. By rescaling the network stiffness $E_{e} \rightarrow E_{e} / w^{2}$ when $w \rightarrow 0$, the network will become a central-force network. Equation (4) then gives $E_{e} \propto w \rightarrow 0$, 
which is consistent with the lack of rigidity for central-force networks. The opposite limit is achived when $q \rightarrow \infty$, which means that $w / \hat{l} \rightarrow \infty$. Equation (4) then gives $E_{e}$ $\propto E w^{2} q / L_{f}$. In the high coverage limit, the stiffness of the network is simply proportional to $E$ multiplied by the density of fiber material in the network. That is, the network behaves as an elastic continuum. Since the network is Poissonian, all density fluctuations vanish at infinite coverage and, consequently, it is quite natural that the network becomes an elastic continuum in this limit.

In Eq. (3) we have, however, assumed that all segments are deformed. Below the critical density of percolation $E_{e}$ is zero, and no segments are deformed because the network is not connected. Above $q_{c}$ there are also segments that carry no load. At high densities such segments only appear at each end of the fibers with a density of $\pi\left(\approx 0.55 q_{c}\right)$ independent of $q$ [4]. The simplest possible transformation from $q$ to the density of loaded fiber segments $\left(q_{l}\right)$ is given by $q / q_{c}$ $=q_{l} / q_{c}+0.55+0.45 /\left(q_{l} / q_{c}+1\right)$. This equation is just a simple crossover from $q=q_{c}$ when $q_{l}=0$, to $q_{l} \rightarrow q$ $-0.55 q_{c}$ in the limit when $q_{l}$ and $q$ approaches infinity. In Eq. (3) we can thus replace the first $q$ on the right-hand side by $q_{l}$,

$$
q_{l}=\frac{q_{c}}{2}\left\{\frac{q}{q_{c}}-1.55+\left[\left(1.55-\frac{q}{q_{c}}\right)^{2}-4\left(1-\frac{q}{q_{c}}\right)\right]^{1.2}\right\} .
$$

With this replacement $E_{e}$ vanishes at $q=q_{c}$, as it should.

In the limits of both a large and a vanishing $q$, Eq. (3) can be written in the form of a series expansion, but for practical purposes it is best to approximate the exponential-integral function by a rational approximation [15]

$$
E_{1}(z)=\frac{z^{2}+a_{1} z+a_{2}}{z^{2}+b_{1} z+b_{2}} \frac{e^{-z}}{z}+\varepsilon(z) \frac{e^{-z}}{z},
$$

where $a_{1}=2.3347, a_{2}=0.2506, b_{1}=3.3307, b_{2}=1.6815$, and $|\varepsilon(z)|<5 \times 10^{-5}$.

If we finally define $z_{l}$ as $z_{l} \equiv 2 q_{l} l_{c} /\left(\pi L_{f}\right)$, and insert it together with Eq. (5) in Eq. (4), we arrive at a rather simple expression for $E_{e}$ as a function of $E, w, q$, and $L_{f}$. A reduced network stiffness $E_{r} \equiv 16 \sqrt{2(1+\nu)} E_{e} /(E w \pi)$ can now be written in a universal one-parameter form,

$$
\begin{aligned}
E_{r}(z)= & z_{l}\left[\frac{z^{2}}{2(1+\nu)}\left(\frac{e^{-z}}{z}-E_{1}(z)\right)\right. \\
& \left.+\left(3+\frac{1}{2(1+\nu)}\right)\left(1-e^{-z}\right)\right] .
\end{aligned}
$$

\section{COMPARISON OF RESULTS}

To test the validity of the effective-medium solution we consider separately all the major ingredients in the model: the exponential length distribution of the segments, the Cox model displacement field $\left[D_{b}=\sin (\theta) \cos (\theta), D_{a}\right.$ $\left.=\cos ^{2}(\theta), \Omega(\theta)=0\right]$, and the assumption that fiber segments are deformed by bending if they are longer than $l_{c}$, and by stretching and shearing if they are shorter than $l_{c}$. In Fig. 2(a) we show the numerical segment-length distributions for $q=4 q_{c}$ and $q=6 q_{c}$ in comparison with Eq. (2). Figure 2(b)
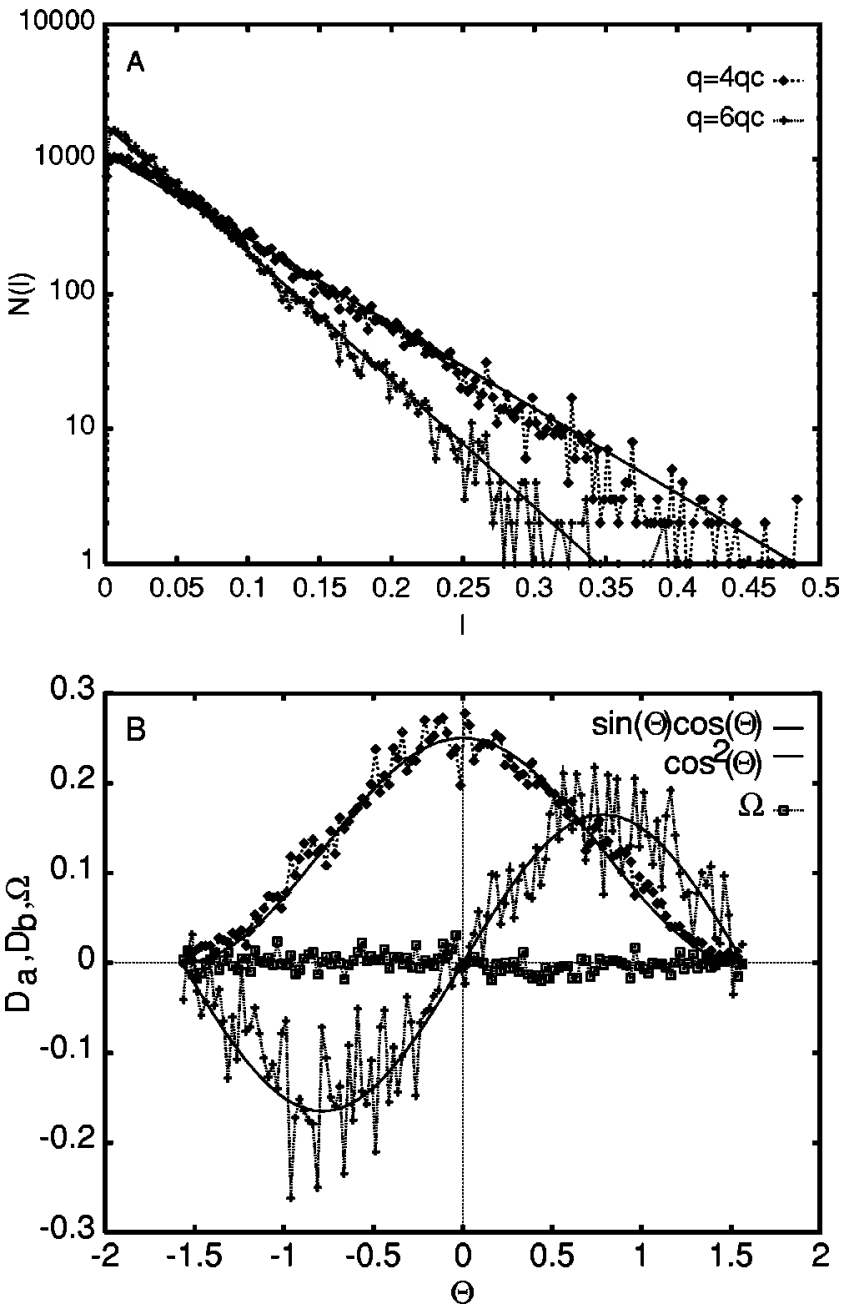

FIG. 2. (a) The segment-length distributions for $q / q_{c}=4$ and $q / q_{c}=6$. The numerical distributions are compared with Eq. (2) (unnormalized). (b) The average transverse (bending) deformations $\left[D_{b}=\sin (\theta) \cos (\theta)\right]$ and the average axial (elongation) deformations $\left[D_{a}=\cos ^{2}(\theta)\right]$, and the average rotation $[\Omega(\theta)]$ as a function of the orientation angle $\theta$.

displays the average orientation distribution of the displacements compared with the Cox model displacement field. In Figs. 3(a) and 3(b) we show the probability distribution of the relative bending deformations $\left[D_{b} /\left(D_{a}+D_{b}\right)\right]$ as a function of the segment length $(l)$. In Fig. 3(a) the fiber width is $w=0.01$, and in Fig. 3(b), $w=0.04$. The assumption of a step-function crossover from $D_{b} /\left(D_{a}+D_{b}\right)=0$ to $D_{b} /\left(D_{a}\right.$ $\left.+D_{b}\right)=1$ at $l_{c}$ is, of course, too simple to be exact, but it nevertheless describes the qualitative behavior of the deformations as evidenced by the plots. Bending deformations dominate when $w=0.01$. It is only for the very shortest segments that pure axial defomation $\left[D_{b} /\left(D_{a}+D_{b}\right)=0\right]$ has a peak in the distribution. For the broader fibers the axialdeformation peak is larger and extends to longer segments. The crossover to bending deformation is also slower than for the slender fibers. Based on this figure one can draw the conclusion that the crossover from pure bending to pure shearing/stretching has a more complicated behavior than the simple step function used in the effective-medium model. To obtain a quantitative agreement between the model solution and the numerical simulations, $l_{c}$ should thus be treated as a 
(A) $w=0.01$
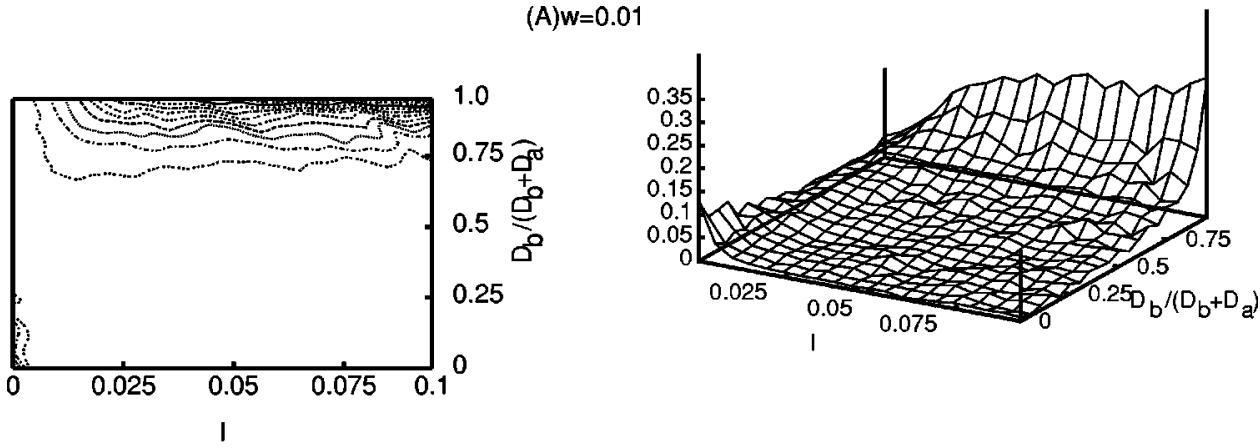

(B) $w=0.04$
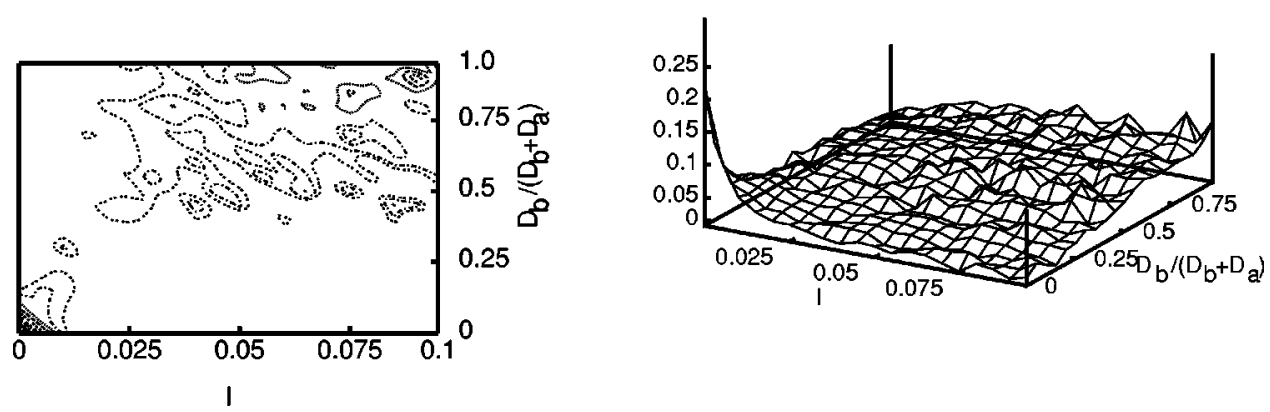

FIG. 3. The probability distribution of the relative bending deformation $\left[D_{b} /\left(D_{b}+D_{a}\right)\right]$ as a function of the fiber segment length $l$. The data are normalized separately for all fiber segment lengths, and are shown both as contours and as surfaces; $w$ $=0.01$ in (a), and $w=0.04$ in (b); $q / q_{c}=4$. fitting parameter. It then plays a role that is rather similar to that of the Debye frequency in the specific heat of lattice phonons. Figures 1-3 nevertheless demonstrate that all the separate assumptions in the effective-medium solution are, to a satisfactory degree, verified by the numerical simulations. The only noteworthy deviation is, as metioned above, the somewhat more complicated behavior of $l_{c}$.

We now continue by comparing the network stiffness of the effective-medium model with numerical simulations. For rather small values of $q$, i.e., for $q \approx 10 q_{c}$ or less, $E_{e}$ is a nonlinear function of $q / q_{c}$. For $q \sim q_{c}, E_{e}$ increases more rapidly than linearly, and for larger $q$ the stiffness approaches a linear dependence on $q$. Earlier computer-simulation results for $q / q_{c}<10$ were fitted with expressions of the form $E_{e}=E_{e f f}\left(q / q_{c}-K\right)[4,5,16]$. The resulting values of $E_{e f f}$ and $K$ from these simulations are in Fig. 4 compared with

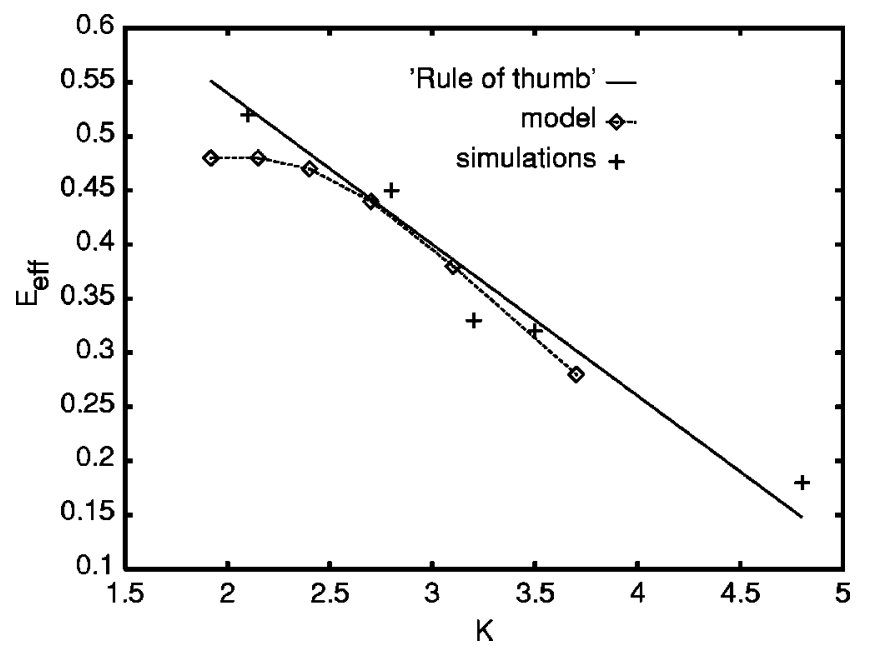

FIG. 4. $E_{\text {eff }}$ as a function of $K$ : the values given by Eq. (4) (model), published finite element method simulations (simulations), and the "rule of thumb" of Ref. [5]. their counterparts as calculated from Eq. (4). Notice that there has been no fitting of any parameters in Fig. 4, which means that the model results and the simulation results from the literature are in excellent agreement. In our model solution, $E_{\text {eff }}$ decreases and $K$ increases a bit faster with increasing $w$ than in the simulations. This is not surprising since, as already noted, a quantitative agreement with the effectivemedium solution and the numerical simulations can only be obtained by fitting $l_{c} \propto w$. One has to notice also that, of the data presented in Fig. 4, only two points with the lowest $K$ value were simulated for an exactly similar setup. The difference lies in the fiber-to-fiber bonds, which were for the other three points taken to be "elastic," that is the fibers were connected with flexible, springlike bond elements. In contrast we have used stiff bonds (see also Sec. II). The two simulations with stiff bonds are for $w=0.06$ and $w=0.01$,

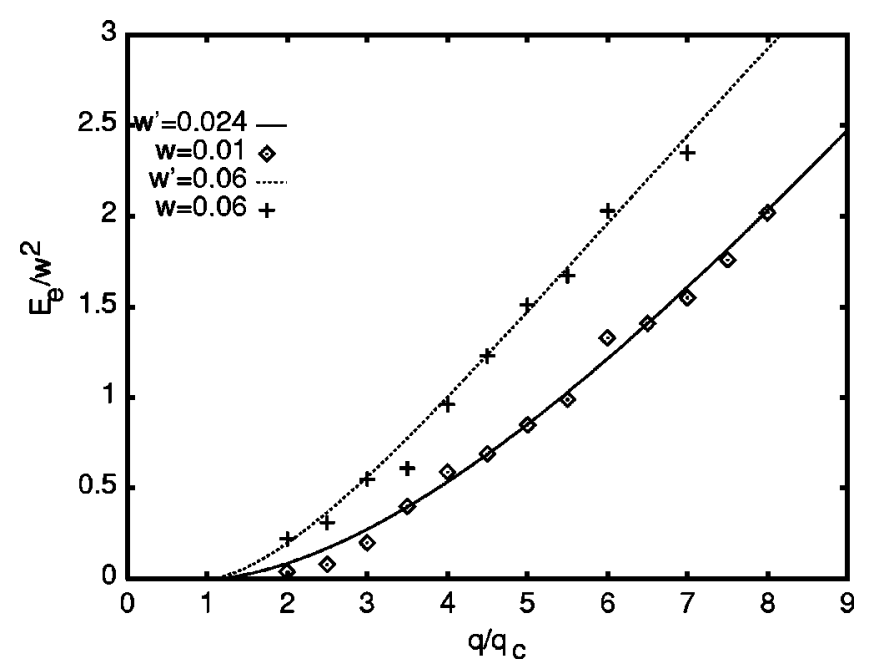

FIG. 5. A comparison of the model results (lines) with $w^{\prime}$ fitted and the simulation results (markers); $w=0.01,0.06$, and $w^{\prime}$ $=0.024,0.06$. 


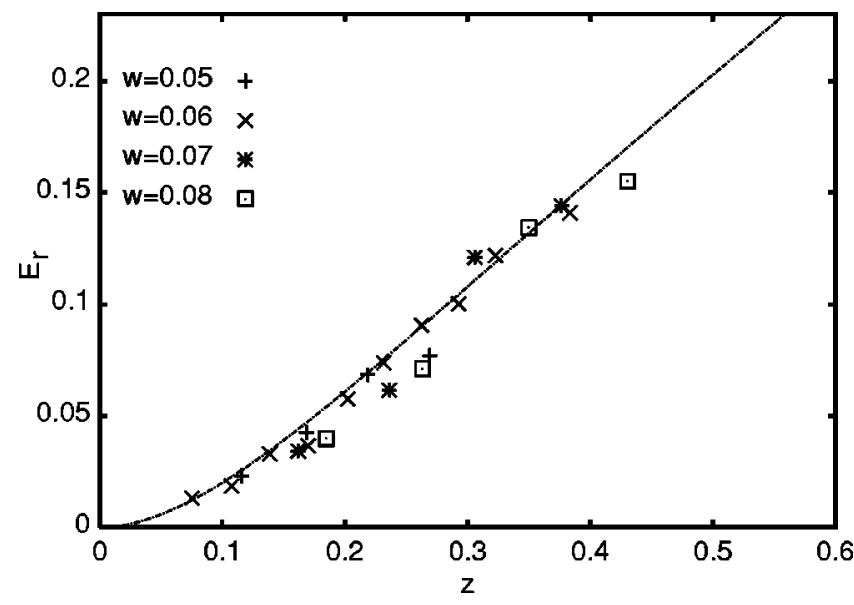

FIG. 6. $E_{r}$ versus $z$ as given by Eq. (7), and simulation results for $w=0.05,0.06,0.07,0.08$.

while the model solutions in Fig. 4 are for $w$ $=0.06,0.05,0.04,0.03,0.02,0.01$, from left to right. In any case, Eq. (4) gives quite correct values for $K$ unlike the shear-lag model which gives too small values [5].

As mentioned above, we modify Eq. (3) by using the integration limit $l_{c}$ as a fitting parameter. In practice we replace $w$ in $l_{c}$ by $w^{\prime}$, and then use the latter to fit the simulation results. The final comparison between the simulation and the model results is given in Fig. 5. The best fits were obtained by $w^{\prime}=0.024$ for $w=0.01$ and by $w^{\prime}=0.06$ for $w$ $=0.06$. By extending the simulations to other $w$, we found that the fitted integration limit $l_{c}$ can be expressed as $l_{c}$ $=(0.68 w+0.016) \sqrt{2(1+\nu)}$. This means that a correction to Eq. (7) is needed for small $w$. For $w>0.04$, however, the universal form seems to work well as is evident from Fig. 6.

The effective-medium model is easily modified to account for random fiber networks which are composed of different types of fibers. The model can furthemore handle changes in the local density of fibers as far as they take place on length scales of the order of single fiber segments. That is, as far as the locations of the segments are completely random, and the texture of the network is completely described by the segment-length distribution. A good example of this kind of extension of the model is that to a variable degree of bonding. It was assumed above that all fiber crossings are bonded. If only a fraction $(a)$ of them were bonded, the segment-length distribution would change to $\sigma(l)=2 a q /\left(\pi L_{f}\right) \exp \left[-2 q /\left(\pi L_{f}\right) a l\right]$, which would modify $z$ and $z_{l}$ and thereby the network stiffness. A comparison of calculated and simulated stiffnesses of networks with a varying bonding probability $a$ is shown in Fig. 7 for $q / q_{c}=3$ and $q / q_{c}=5$, and $w=0.01, w=0.06$. Obviously there is a fairly good agreement between the two sets of results.

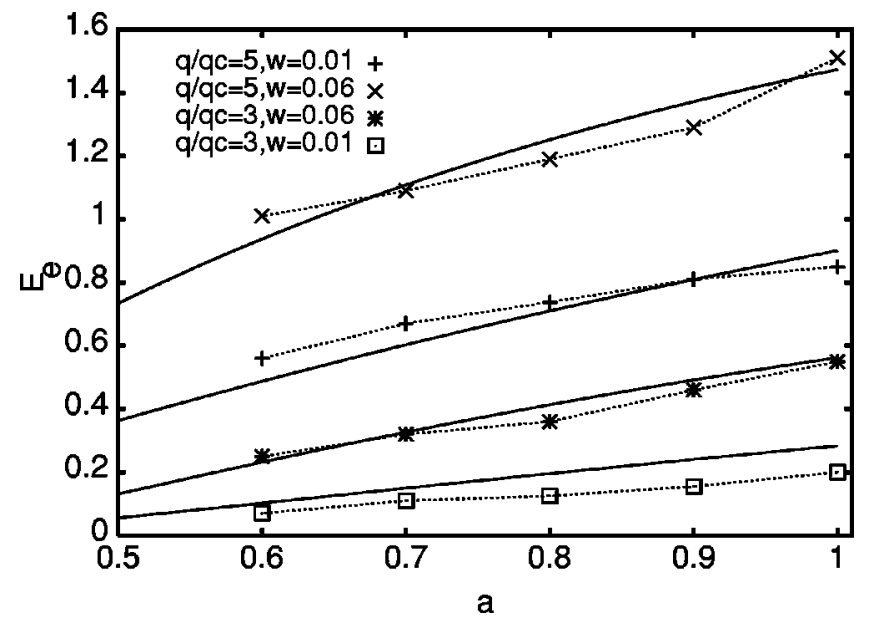

FIG. 7. $E_{e}$ versus bonding probability $a ; q / q_{c}=3,5$, and $w$ $=0.01,0.06$.

\section{SUMMARY}

The description obtained for random fiber networks with the effective-medium theory presented here can also be compared with experiments. By measuring the tensile modulus of paper made from pulp fibers of different lengths, it has been found [10] that the stiffness of paper sheets is of the form $E_{e}(\rho)=A\left(\rho-\rho_{0}\right)$, where $\rho \equiv w^{2} q / L_{f}$, and $A$ and $\rho_{0}$ are constants. In contrast with this, the Cox or the shear-lag model gives a stiffness of the form $E_{e}(\rho)=B\left(\rho-\rho^{\prime} / L_{f}\right)$, where $\rho^{\prime}$ is a constant. Equation (4) is also nearly linear, except close to $q=q_{c}$ (cf. Figs. 5 and 6). Replacing $q$ with $\rho$ in the definition of $z$ gives $z=2 l_{c} \rho /\left(\pi w^{2}\right)$, which is obviously independent of $L_{f}$. We find, therefore, that Eq. (4) is consistent with experiments with respect to changes in $L_{f}$, unlike the Cox and shear-lag models.

We can conclude by summarizing the main results in a more general form. We have introduced a modified effectivemedium model for the elasticity of inherently heterogeneous materials. It is based on using the bonds that are formed between the points where the building blocks of the material are joined together as the basic variables. That is, the microscopic geometry of the networks, for which statistical properties are often available, is taken into account. The model is tested on two-dimensional random fiber networks and is found to work reasonably well. This means that the behavior of the model agrees well with that found from numerical simulations and also that discrepancies between theory and experiments could be avoided and explained. The model can act as a theoretical foundation for investigating, e.g., changes in the tensile modulus as the texture of a heterogeneous material is altered.

\section{ACKNOWLEDGMENTS}

We acknowledge discussions with K. Niskanen and support by the Academy of Finland.
[1] S. Torquato, Appl. Mech. Rev. 44, 37 (1991).

[2] I. Balberg and N. Binenbaum, Phys. Rev. B 28, 3799 (1983); M. Deng and C. T. J. Dodson, Paper, an Engineered Stochastic Structure (Tappi Press, Atlanta, 1994).

[3] K. J. Niskanen and M. J. Alava, Phys. Rev. Lett. 73, 3475
(1994); N. Provatas, T. Ala-Nissilä, and M. J. Alava, ibid. 75, 3556 (1995).

[4] J. Åström, S. Saarinen, and J. Kurkijärvi, J. Appl. Phys. 75, 2383 (1994); J. A. Åström and K. J. Niskanen, Europhys. Lett. 21, 557 (1993). 
[5] V. I. Räisänen, M. J. Alava, K. J. Niskanen, and R. M. Nieminen, J. Mater. Res. 12, 2725 (1997).

[6] M. Murat, M. Anholt, and H. D. Wagner, J. Mater. Res. 7, 3120 (1992).

[7] L. Monette, M. P. Anderson, and G. S. Grest, J. Appl. Phys. 75, 1155 (1994).

[8] M. Kellomäki, J. Aström, and J. Timonen, Phys. Rev. Lett. 77, 2730 (1996).

[9] M. Sahimi, Phys. Rep. 306, 213 (1998).

[10] M. Kimura and H. Uchimura, Sen'i Gakkaishi 51, 550 (1995).
[11] H. L. Cox, Br. J. Appl. Phys. 3, 72 (1952).

[12] D. H. Page and R. S. Seth, Tappi J. 63, 113 (1980).

[13] G. E. Pike and C. H. Seager, Phys. Rev. B 10, 1421 (1974).

[14] D. J. Jacobs and M. F. Thorpe, Phys. Rev. Lett. 75, 4051 (1995); C. Moukarzel and P. M. Duxbury, ibid. 75, 4055 (1995).

[15] Handbook of Mathematical Functions, edited by M. Abramowitz and I. A. Stegun, (Dover, New York, 1972).

[16] A. Jangmalm and S. Östlund, Nordic Pulp Pap. Res. J. 10, 156 (1995). 